\title{
Comunicación
}

\section{Hemangiosarcoma Cardiaco Primario en Aurícula Derecha en un Canino Rottweiler de Ocho Años de Edad}

\author{
Canine Primary Cardiac Hemangiosarcoma of Right Atrium in a Rottweiler \\ of Eight Years OLd
}

\author{
Eben Salinas C., ${ }^{1,3}$, Roberto Dávila F., ${ }^{2,4}$ Edith Chávez R. ${ }^{1}$
}

\section{Resumen}

\begin{abstract}
El hemangiosarcoma es un tumor maligno de origen vascular y es el más frecuente de los tumores cardiacos de los canes. Se presenta el caso de un perro de raza Rottweiler de ocho años de edad, que presentó disnea, mucosas pálidas e intolerancia al ejercicio. A la auscultación cardiaca, los sonidos fueron levemente audibles y el pulso femoral disminuido. Los exámenes de laboratorio evidenciaron trombocitopenia e insuficiencia renal aguda. Se realizaron radiografías y ecografías, diagnosticándose hidropericardio y taponamiento cardiaco. Se realizó una pericardiocentesis, cuyo estudio citológico fue compatible con exudado serosanguinolento. La tomografía axial computarizada del tórax solo identificó aumento de tamaño de la aurícula derecha. Se realizó una toracotomía lateral derecha, identificándose una masa de tipo neoplásica en la aurícula derecha, así como nódulos metastásicos en pericardio y pulmones. El estudio histopatológico diagnosticó un hemangiosarcoma cardiaco primario en aurícula derecha de tipo cavernoso.
\end{abstract}

Palabras clave: hemangiosarcoma; Rottweiler; toracotomía; taponamiento; tomografía

\section{Abstract}

Haemangiosarcoma is a malignant tumour of vascular origin and it is the most frequent of cardiac tumours in dogs. It is presented the case of a 8-year old Rottweiler dog with dyspnea, pale mucous and intolerance to exercise. Cardiac sounds were slightly perceptible with decreased femoral pulse. Lab tests showed thrombocytopenia, and acute kidney failure. Radiographic and echography tests were carried out identifying only pericardial

${ }^{1}$ Clínica Veterinaria Doctor Pet's, Lima, Perú

${ }^{2}$ Clínica de Animales Menores, Facultad de Medicina Veterinaria, Universidad Nacional Mayor de San Marcos, Lima, Perú

${ }^{2}$ E-mail: maxteria@yahoo.com

${ }^{4}$ E-mail:rodaf45@yahoo.com

Recibido: 3 de febrero de 2017

Aceptado para publicación: 26 de mayo de 2017 
effusion and cardiac obstruction. Pericardiocentesis was performed which cytology study was compatible with bloodstained serum exudate. A computerized axial tomography in thorax only detected enlargement of the right atrium. A right lateral thoracotomy was performed, identifying a neoplastic mass in the right atrium and several metastatic nodules in lungs and pericardium. The histopathological study diagnosed a primary cardiac hemangiosarcoma in the right atrium of cavernous type.

Key words: haemangiosarcoma; Rotweiller; thoracotomy; obstruction; tomography

\section{INTRODUCCIÓN}

El hemangiosarcoma (HSA) es una neoplasia maligna de origen vascular endotelial, también conocida como hemangioendotelioma maligno (Dahl et al., 2008). En el perro, su presentación alcanza el 7\% de las neoplasias malignas primarias no cutáneas y entre el 12 y $21 \%$ de las neoplasias de origen mesenquimal (Thamm, 2007). El HSA representa el $69 \%$ de los tumores cardiacos y se presenta principalmente en perros de edad avanzada (8-13 años). Entre las razas más predispuestas se encuentran el Pastor Alemán, Golden Retriever y Labrador Retriever; sin embargo, puede presentarse en cualquier grupo racial (Fry et al., 2014).

En el HSA se manifiestan comúnmente alteraciones como anemia y trombocitopenia, a consecuencia de la infiltración progresiva de células neoplásicas en la médula ósea, llegando a ocurrir trombosis multisistémica por coagulación intravascular diseminada (CID) (Mac Ewen, 2001). Este síndrome finalmente produce hemorragias espontáneas, shock y muerte.

El HSA cardiaco primario o metastásico se ubica preferentemente en la aurícula o atrio derecho, presentándose como una masa única o como estructuras difusas no organizadas. Estas masas pueden erosionar y producir la ruptura de las paredes del miocardio, conduciendo a un hidropericardio y taponamiento cardiaco con signos de insuficiencia cardiaca derecha (Treggiari et al., 2015). Las radiografías de tórax generalmente tienen poca sensibilidad para identificar masas cardiacas. Radiográficamente, en un hidropericardio se podrá observar, básicamente, aumento de la silueta cardiaca debido a la efusión pericárdica (Holt et al., 1992). La ecocardiografía bidimensional 2D muestra relativa sensibilidad para identificar neoplasias cardiacas, aunque es el método más confiable para detectar hidropericardio, apoyando en el diagnóstico del taponamiento cardiaco (Rajagopalan et al., 2013).

No se tiene un tratamiento efectivo para el HSA. El manejo del paciente se sustenta en la mejora de la calidad de vida mediante quimioterapia o cirugía. Los tiempos de sobrevida son cortos, ya que se consideran la existencia de micro-metástasis una vez diagnosticado el tumor primario (Dahl et al., 2008).

\section{Caso Clínico}

Un perro macho de raza Rottweiler, de 8 años de edad y $40 \mathrm{~kg}$ de peso, se presenta a consulta por un cuadro de disnea, mucosas pálidas e intolerancia al ejercicio. Al examen físico, el paciente presentaba una condición corporal de $2 / 5$, frecuencia respiratoria y cardiaca aumentada y un grado de deshidratación del 5\%. A la auscultación del corazón, los sonidos fueron levemente audibles con un pulso femoral disminuido. 
Cuadro 1. Valores del hemograma y bioquímica de un perro Rottweiler, macho, de 8 años de edad, presentado a consulta por un cuadro de disnea, mucosas pálidas e intolerancia al ejercicio

\begin{tabular}{lcc}
\hline Parámetro & Resultado & Valores de referencia $^{1}$ \\
\hline Glóbulos rojos $\left(\mathrm{x} 10^{6} / \mu \mathrm{l}\right)$ & 6180 & $5.5-8.5$ \\
Hematocrito $(\%)$ & 39.1 & $37-55$ \\
Hemoglobina $(\mathrm{g} / \mathrm{dl})$ & 13.7 & $12-18$ \\
Recuento de glóbulos blancos $\left(\mathrm{x} 10^{3} / \mu \mathrm{l}\right)$ & 9.82 & $6-17$ \\
$\quad$ Neutrófilos segmentados $(\%)$ & 64.5 & $60-77$ \\
$\quad$ Linfocitos $(\%)$ & 17.4 & $12-30$ \\
Monocitos $(\%)$ & 6.9 & $3-10$ \\
$\quad$ Eosinófilos $(\%)$ & 11.2 & $2-10$ \\
Plaquetas (x10 $/ \mathrm{ul})$ & 209 & $200-500$ \\
Alanina amino transferasa (UI/L) & 70 & $<40$ \\
Aspartato aminotransferasa (UI/L) & 127 & $<60$ \\
Creatinina (mg/dl) & 4.0 & $20-156$ \\
Fosfatasa Alcalina & 91 & $20-40$ \\
Urea (mg/dl) & 130 & \\
\hline
\end{tabular}

${ }^{1}$ Kahn (2007)

Se solicitaron pruebas hematológicas, bioquímica sanguínea completa y de orina. En el hemograma, las plaquetas estaban en el límite inferior del intervalo de referencia (Cuadro 1). La bioquímica sanguínea mostró un incremento en los valores de urea y creatinina, así como en los niveles de alanina aminotransferasa (Cuadro 1). En el uroanálisis se encontró proteinuria $(100 \mathrm{mg} / \mathrm{dl})$. Estos hallazgos indicaron signos de insuficiencia renal e insuficiencia hepática.

Se realizó una ecografía abdominal, identificándose moderada congestión hepática, aunque el bazo, mesenterio y otras vísceras intestinales no mostraron alteraciones ecográficas. En las placas radiográficas de tórax se identificó aumento de tamaño de las cámaras del corazón, compatible con una cardiomegalia global sugerente de hidropericardio. En la ecocardiografía bidimen- sional 2D se observó acumulación de líquido en el saco pericárdico, de característica anecoica, que producía colapso del ventrículo $\mathrm{y}$ atrio derechos. No se evidenciaron masas neoplásicas de origen cardiaco.

Se planteó el diagnóstico presuntivo de taponamiento cardiaco sin causa definida, pero sin descartar la etiología neoplásica. Se realizó una pericardiocentesis ecoguiada, extrayéndose $500 \mathrm{ml}$ de líquido pericárdico turbio rojizo que no coaguló a los 15 minutos. Se realizaron estudios citológicos y bacteriológicos de esta muestra, resultando compatible con exudado serosanguinolento. Complementariamente, se realizó una tomografía axial computarizada (TAC) del tórax en cortes axiales de $5 \mathrm{~mm}$, sin que se identifiquen masas de tipo neoplásico en corazón ni en pulmones (Figura 1). 

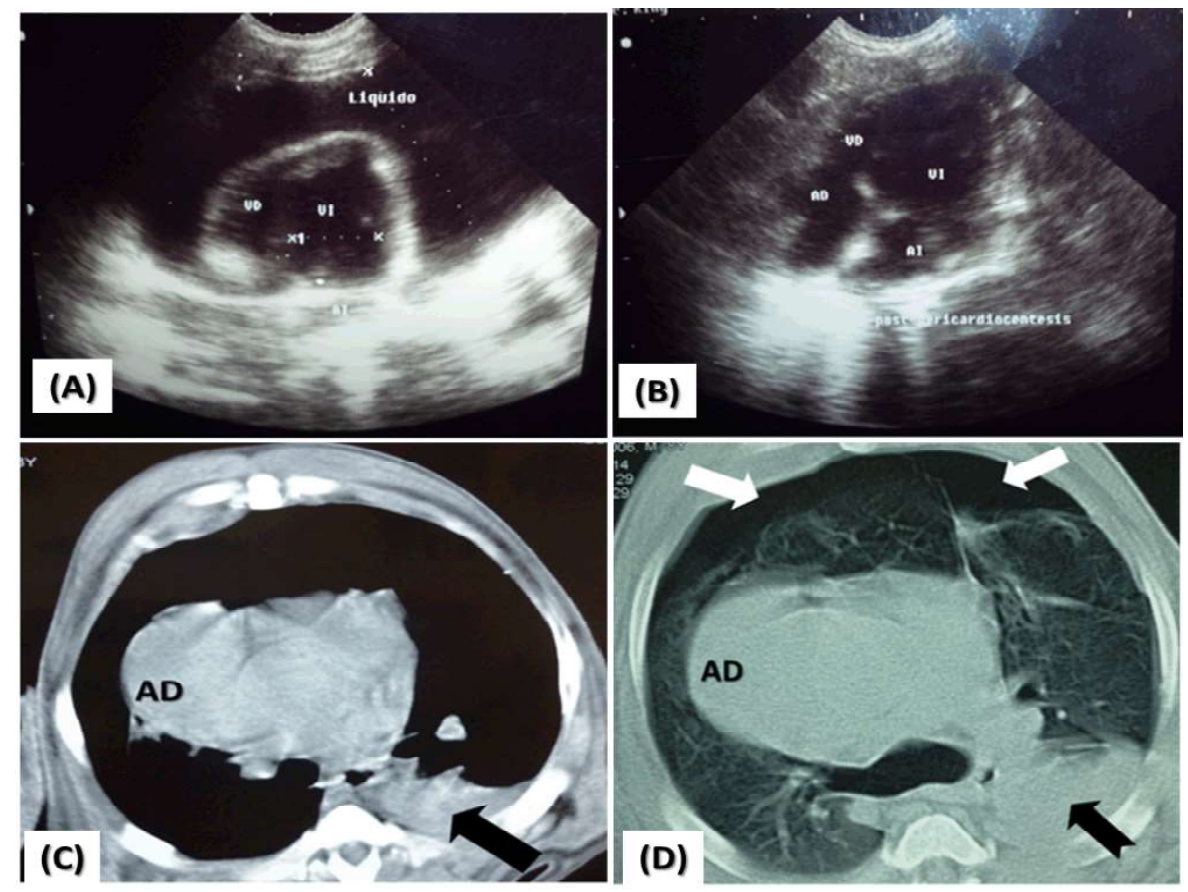

Figura 1. Ecocardiografía 2D y tomografía computarizada en un perro Rottweiler, macho, de 8 años de edad. (A): Líquido libre en saco pericárdico con colapso de aurícula y ventrículo derechos; (B) Imagen cardiaca pos-pericardiocentesis; (C) Tomografía axial computarizada (TAC) con ventana mediastínica: incremento de tamaño de la aurícula derecha (AD), efusión pleural en hemitórax izquierdo (flecha negra); (D) TAC con ventana pulmonar: incremento de tamaño de aurícula derecha (AD), neumotórax bilateral (flechas blancas) y efusión pleural (flechas negras)

\section{Toracotomía Intercostal}

Debido a la inconsistencia de los resultados de laboratorio e imágenes, se sugirió a los propietarios la exploración quirúrgica del tórax y realizar pericardiectomía como tratamiento inicial del hidropericardio. Como medicación pre-anestésica, se administró morfina $0.5 \mathrm{mg} / \mathrm{kg}$, midazolán $0.2 \mathrm{mg} / \mathrm{kg}$, cefalotina $30 \mathrm{mg} / \mathrm{kg}$, vía endovenosa, y fentanilo en infusión continua a $5 \mu \mathrm{g} / \mathrm{kg} / \mathrm{h}$. Para la inducción se usó propofol $5 \mathrm{mg} / \mathrm{kg}$ intravenoso. El mantenimiento anestésico se realizó con isofluorano en oxígeno con un circuito de no reinhalación, realizándose ventilación asistida durante todo el proceso quirúrgico.
Luego de la preparación del campo quirúrgico en asepsia y antisepsia, se practicó una incisión sobre la piel a nivel del quinto espacio intercostal derecho y se diseccionó los planos subcutáneos y musculares. Para ingresar a la cavidad torácica se realizó una inciso-punción en los músculos intercostales y la pleura parietal. Luego, se ingresó al espacio intercostal usando un separador autoestático de Finochietto (Fossum, 2008).

En la exploración de la cavidad torácica, se identificó moderada cantidad de líquido libre sero-sanguinolento y signos de hepatización pulmonar difusa. El saco pericárdico tenía apariencia hemorrágica, $\mathrm{y}$ 

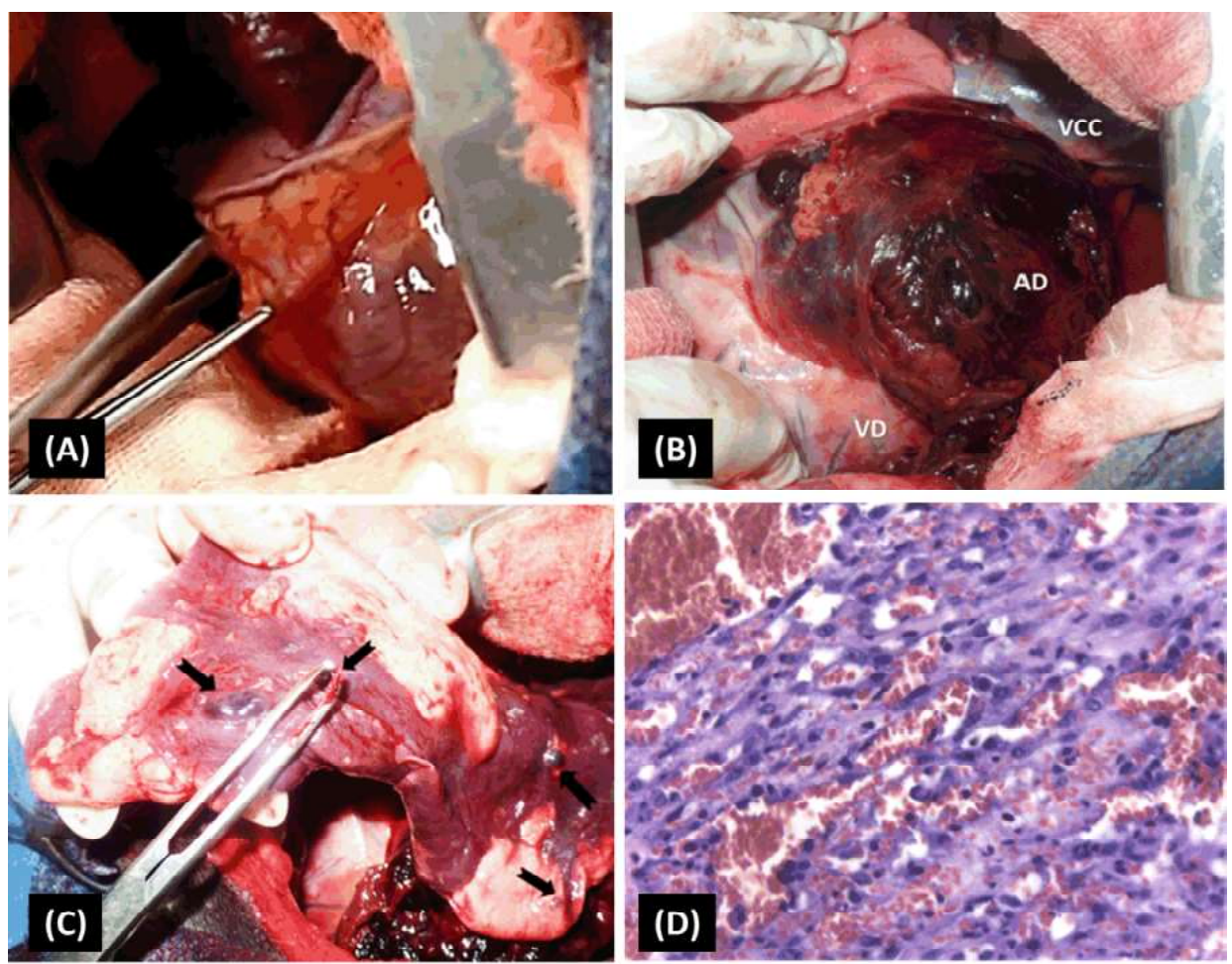

Figura 2. Toracotomía en un perro Rottweiler, macho, de 8 años de edad. (A) Pericardio engrosado y vascularizado; (B) Masa neoplásica en aurícula derecha (AD); (C) Lóbulo pulmonar con hepatización y nódulos tumorales metastásicos (pinza y flechas); (D) Histopatología: áreas de hemorragia en nidos de células endoteliales neoplásicas con alto pleomorfismo celular, límites citoplasmáticos indistintos y dispuestos en haces de entrelazado encerrando espacios llenos de sangre (H\&E, 40x). (VD) ventrículo derecho, (VCC) vena cava caudal

contenía una pequeña cantidad de líquido sanguinolento. Se procedió a realizar una pericardiectomía subtotal subfrénica, realizándose una incisión en forma de $\mathrm{T}$ en el pericardio a lo largo de la base cardiaca, previa localización del nervio frénico derecho, que sirvió como límite para la exéresis del pericardio (Orton, 2006). Una vez retirado el pericardio, se identificó una masa de tipo neoplásica no encapsulada de color negro rojizo y que abarcaba todo el diámetro de la aurícula derecha. Se encontraron focos de infiltración neoplásica en la cara interna del pericardio y en el parénquima pulmonar adyacente. Debido a la irregularidad e invasión de la masa neoplásica sobre la aurícula derecha, pericardio y lóbulos pulmonares, se realizó una citorreducción tumoral y toma de muestras del tejido neoplásico para llegar al diagnóstico definitivo mediante el estudio histopatológico.

Se procedió al cierre de la toracotomía, colocando ocho suturas monofilamento no absorbible rodeando las costillas adyacentes a la incisión para lograr aproximarlas y reponer así el espacio intercostal. Se colocó una sonda para drenaje torácico, ubicándolo a dos espacios intercostales caudales a la incisión y se acopló a una llave de tres vías para realizar aspiración intermitente. Se realizaron la síntesis de los planos musculares, tejido subcutáneo y piel de forma habitual. Finalmente, se hicieron aspiraciones del aire residual, confirmándose la no presentación de neumotórax. 
El paciente pasó a recuperación anestésica y manejo del dolor posquirúrgico con opioides sistémicos (morfina $0.5 \mathrm{mg} / \mathrm{kg}$ $\mathrm{c} / 8 \mathrm{~h}$, vía EV), antiinflamatorios (meloxican $0.2 \mathrm{mg} / \mathrm{kg}$, c/24h, vía IM), además de anestésicos locales (lidocaína $2 \%$, vía intracavitario); sin embargo, falleció a las 12 horas. Los propietarios no accedieron a la necropsia al paciente. El diagnóstico histopatológico de las muestras remitidas fue de hemangiosarcoma cardiaco de tipo cavernoso en atrio derecho y pericardio (Figura 2).

\section{Discusión}

El paciente desarrolló múltiples alteraciones orgánicas a consecuencia de la falla cardiaca derecha, así como del HSA. Los resultados de la bioquímica sanguínea fueron compatibles con un cuadro de insuficiencia renal y hepática aguda. Los mecanismos que empeoran la función renal en pacientes cardiópatas, como la hipotensión renal y el bajo volumen minuto, conllevan a la disminución de la función renal por disminución del filtrado glomerular (Belziti, 2010). El recuento de plaquetas estaba en el límite inferior del intervalo de referencia, lo cual sugirió un estado crónico de coagulación intravascular diseminada (CID). El paciente presentó pocas manifestaciones clínicas esperadas de este síndrome, aunque está reportada la prevalencia de la trombosis sobre la hemorragia (Bick, 1988; Nelson y Couto, 2010).

Los estudios por imágenes no fueron concluyentes en apoyo del diagnóstico del HSA cardiaco. El mayor beneficio de los rayos $\mathrm{X}$ de tórax en los pacientes con neoplasias cardiacas está en determinar la presencia de metástasis pulmonares, efusión pleural y neumotórax (Côté et al., 2013). Asimismo, la ecocardiografía bidimensional 2D en este paciente presentó baja precisión para el diagnóstico del HSA en aurícula derecha. MacDonald et al. (2009) y Yamamoto et al. (2013) han reportado la baja sensibilidad de la ecocardiografía para confirmar masas neoplásicas en aurícula derecha y pericardio (17 y $82 \%$, respectivamente). Los estudios por TAC de tórax tampoco fueron determinantes para identificar masas de tipo neoplásico en corazón. Estos hallazgos coinciden con Scollan et al. (2015), donde la TAC no mejoró la detección de masas cardiacas en perros con efusión pericárdica sobre la ecocardiografía. Una de las razones que dificultan la identificación de una masa neoplásica cardiaca suele ser la irregularidad de las estructuras, el tamaño y la localización del tumor en aurícula o atrio derecho. Estos resultados coinciden con lo presentado en el caso clínico, dado que se necesitó la exploración quirúrgica y el estudio histopatológico para tener el diagnóstico definitivo.

El tratamiento de la efusión pericárdica y taponamiento cardiaco es la pericardiocentesis. Esta maniobra tiene por objetivo disminuir la presión intrapericárdica para restablecer la hemodinámica del sistema cardiovascular (Tobias, 2005). El estudio citológico del líquido drenado fue compatible con exudado serosanguinolento. La citología tiene un bajo aporte al diagnóstico del HSA y otros tumores por su baja capacidad de exfoliar células neoplásicas (Cagle et al., 2014).

En el paciente, la cirugía se realizó con dos objetivos: en primer lugar, para tratar la efusión pericárdica y evitar el taponamiento cardiaco mediante una pericardiectomía subfrénica; y en segundo lugar, para realizar una exploración quirúrgica del tórax que lleve a un diagnóstico definitivo (Tobias y McNiel, 2008). La identificación de la masa tumoral solo fue posible con la exploración quirúrgica cardiaca; sin embargo, no fue posible realizar la exéresis completa de la masa tumoral en la aurícula derecha debido a la infiltración al miocardio y grandes vasos, de allí que se tonó la decisión de realizar una citorreducción tumoral para contribuir al mejor funcionamiento cardiaco.

La citorreducción tumoral es una alternativa quirúrgica usada en múltiples casos, como en el manejo de tumores ginecológicos 
en seres humanos; logrando una mejora en la supervivencia global (Lee et al., 2015). Se considera que la citorreducción estimularía la fracción de crecimiento de las células tumorales, aumentando la vascularización sanguínea y sensibilizándolas más a la quimioterapia y radioterapia (Kirk y Bonagura, 1999).

El diagnóstico final del HSA se realizó mediante el estudio histopatológico de la lesión. El pronóstico y la supervivencia de los pacientes con HSA cardiaco son bajos, debido principalmente a las metástasis y los síndromes paraneoplásicos. La cirugía es solo un tratamiento paliativo y se considera un tiempo de sobrevida de 122 días (Smith, 2003). La supervivencia está directamente relacionada con la edad del animal en el momento del diagnóstico, disminuyendo la expectativa de vida conforme avanza la edad del paciente (Dahl et al., 2008).

\section{Literatura Citada}

1. Belziti C, Bagnati R, Ledesma P, Vulcano N, Fernández S. 2010. Empeoramiento de la función renal en pacientes hospitalizados por insuficiencia cardiaca aguda descompensada: incidencia, predictores y valor pronóstico. Rev Esp Cardiol 63: 294-302. doi: 10.1016/ S0300-8932(10)70088-9

2. Bick RL. 1988. Disseminated intravascular coagulation and related syndromes: a clinical review. Semin Thromb Hemost 14: 299-338. doi: 10.1055/s-20071002793

3. Cagle LA, Epstein SE, Owens SD, Mellema MS, Hopper K, Burton AG. 2014. Diagnostic yield of cytologic analysis of pericardial effusion in dogs. J Vet Intern Med 28: 66-71. doi: 10.1111/ jvim. 12253

4. Côté E, Schwarz LA, Sithole F. 2013. Thoracic radiographic findings for dogs with cardiac tamponade attributable to pericardial effusion. J Am Vet Med Assoc 243: 232-235. doi: 10.2460/ javma.243.2.232
5. Dahl K, Gamlem H, Tverdal A, Glattre E, Moe L. 2008. Canine vascular neoplasia-A population-based study of prognosis. APMIS Suppl 125: 55-62. doi: 10.1111/j.1600-0463.2008.$125 \mathrm{~m} 5 . \mathrm{x}$

6. Fossum $T W$. 2008. Cirugía del sistema cardiovascular. En: Cirugía de pequeños animales. $3^{a}$ ed. España: Elsevier. p 775816.

7. Fry JK, Burney D, Hottinger H, Fabiani M, Feagin C. 2014. Pollakiuria and stranguria in a Labrador retriever with penile HSA. J Am Anim Hosp Assoc 50: 141-147. doi: 10.5326/ JAAHA-MS-5984

8. Holt D, Van Winkle T, Schelling C, Prymak C. 1992. Correlation between thoracic radiographs and postmortem findings in dogs with hemangiosarcoma: 77 cases (1984-1989). J Am Vet Med Assoc 200: 1535-1539.

9. Kahn C. 2007. El manual Merck de veterinaria. $6^{\mathrm{a}}$ ed. España: Océano. 2682 p.

10. Kirk W, Bonagura J. 1999. Terapéutica veterinaria de pequeños animales. $12^{\circ}$ ed. México: McGraw-Hill Interamericana. $610 \mathrm{p}$.

11. Lee CK, Lord S, Grunewald T, Gebski V, Hardy-Bessard AC, Sehouli J, Woie K, Heywood M, et al. 2015. Impact of secondary cytoreductive surgery on survival in patients with platinum sensitive recurrent ovarian cancer: analysis of the calypso trial. Gynecol Oncol 136: 18-24. doi: 10.1016/j.ygyno.2014.09.017

12. Mac Ewen EG. 2001. Miscellaneus tumours. In: Withrow SJ, MacEwen EG (eds). Small animal clinical oncology. USA: WB Saunders. p 639-667.

13. MacDonald KA, Cagney O, Magne ML. 2009. Echocardiographic and clinicopathologic characterization of pericardial effusion in dogs: 107 cases (1985-2006). J Am Vet Med Assoc 235: 1456-1461. doi: 10.2460/javma.235.12.1456

14. Nelson R, Couto C. 2010. Medicina interna de pequeños animales. España: Elsevier. $1467 \mathrm{p}$. 
15. Orton EC. 2006. Cirugía cardiaca. En: Slatter D, Hosgood G, Holmberg D (eds). Tratado de cirugía en pequeños animales. $3^{a}$ ed. Argentina: Intermédica. $p$ 1119-1153.

16. Rajagopalan $V$, Jesty $S A$, Craig LE, Gompf R. 2013. Comparison of presumptive echocardiographic and definitive diagnoses of cardiac tumors in dogs. J Vet Intern Med 27: 1092-1096. doi: $10.1111 /$ jvim. 12134

17. Scollan KF, Bottorff B, StiegerVanegas S, Nemanic S, Sisson D. 2015. Use of multidetector computed tomography in the assessment of dogs with pericardial effusion. J Vet Intern Med 29: 79-87. doi: 10.1111/jvim. 12479

18. Smith AN. 2003. Hemangiosarcoma in dog and cats. Vet Clin North Am Small Anim Pract 33: 534-547.

19. Thamm DH. 2007. Hemangiosarcoma. En: Withrow SW, Vail DM (eds). Small animal clinical oncology. $4^{\text {th }}$ ed. St. Louis, Missouri: Saunders Elsevier. p 785-795.
20. Tobias AH, McNiel EA. 2008. Pericardial disorders and cardiac tumors. In: Tilley LP, Smith FWK Jr, Oyama MA, Sleeper MM (eds). Manual of canine and feline cardiology. $4^{\text {th }}$ ed. St. Louis, Missouri: Saunders Elsevier. p 205-210.

21. Tobias AH. 2005. Pericardial disorders. In: Ettinger SJ, Feldman EC (eds). Textbook of veterinary internal medicine. $6^{\text {th }}$ ed. St Louis, MO: Saunders. $p$ 1107-1108.

22. Treggiari E, Pedro B, Dukes-McEwan J, Gelzer AR, Blackwood L. 2015. A descriptive review of cardiac tumours in dogs and cats. Vet Comp Oncol 15: 273288. doi: 10.1111/vco.12167

23. Yamamoto S, Hoshi K, Hirakawa A, Chimura S, Kobayashi M, Machida N. 2013. Epidemiological, clinical and pathological features of primary cardiac hemangiosarcoma in dogs: a review of 51 cases. J Vet Med Sci 75: 1433-1441. doi: 10.1292/jvms.13-0064 